\title{
Desarrollo de sistemas mecatrónicos de bajo coste para equipamiento experimental de prácticas docentes
}

\author{
G. Olivares, A.González, F. Gómez, M. Damas, A.Olivares. \\ Departamento de Arquitectura y Tecnología de Computadores. ETSI Informática y de \\ Telecomunicación. Universidad de Granada \\ Granada, España \\ \{gonzalo, mdamas, frgomez, aolivares\}@ugr.es
}

\begin{abstract}
Resumen. Este trabajo describe los objetivos y el estado de desarrollo actual de un conjunto de maquetas y sistemas mecatrónicos de muy bajo coste destinados al aprendizaje de las principales técnicas de control. Se está construyendo un laboratorio con material docente experimental realizado íntegramente por profesores y alumnos, y sin duda será muy útil para dar soporte a la mayoría de las asignaturas relacionadas con sistemas de control impartidas por el Área de Ingeniería de Sistemas y Automática y el Departamento de Arquitectura y Tecnología de Computadores de la Universidad de Granada. El trabajo realizado forma parte del proyecto de innovación docente: "Nuevas experiencias de control óptimo con sistemas mecatrónicos".
\end{abstract}

Palabras Claves: Control, Mecatrónica, Bajo Coste, Docencia Experimental.

\begin{abstract}
This paper describes the objectives and the current state of development of a set of low cost models and mechatronics systems for learning the main control techniques. The control laboratory is being making entirely by teachers and students, and will certainly be very useful to support most of the subjects related control systems, teached by the Engineering Systems and Automation Area and by the Department of Architecture and Technology Computer at the University of Granada. The work is part of teaching innovation project: "New experiences optimal control with mechatronic systems."
\end{abstract}

Keywords: Control, Mechatronics, Low-cost, Experimental Learning.

\section{Introducción}

Con motivo del proceso de implantación de asignaturas del Área de Ingeniería de Sistemas y Automática en los nuevos grados de ingeniería que se imparten en la Escuela Técnica Superior de Ingeniería Informática y de Telecomunicación y en la Facultad de Ciencias de la Universidad de Granada, se han venido preparando en los últimos años un conjunto de prácticas, basadas principalmente en herramientas gráficas de simulación por software. En ese sentido, se han realizando un conjunto de maquetas virtuales para prácticas de control secuencial con PLC (Controladores Lógicos Programables), con magníficos resultados docentes y gran aceptación por 
parte de los alumnos [1,2,3]. Se optó por la simulación virtual principalmente por la facilidad de uso, el bajo mantenimiento y la robustez de la solución, pero además también por la falta de presupuesto y la necesidad de repartir la escasa dotación económica anual entre todas las asignaturas, que hubo que implantar de forma casi simultánea.

Además de la realización de prácticas virtuales simuladas que reducen la necesidad de disponer de un mayor número de maquetas "reales", se considera que es también muy importante disponer de una mayor variedad de dichas maquetas para poder realizar un mayor número de prácticas diferentes. A pesar de las dificultades presupuestarias, cada año se podrían haber adquirido uno o dos productos de empresas especializadas en material de prácticas. Sin embargo, se ha preferido ir preparando con los alumnos, mediante trabajos en grupos y trabajos de fin de Grado y de Master, el diseño inicial de un conjunto de sistemas de control de maquetas mecánicas con control y regulación digital, e ir construyendo poco a poco todo el equipamiento del laboratorio de Ingeniería de Sistemas, Automática y Mecatrónica.

En la mayoría de los centros docentes universitarios se establecen diversos puestos de prácticas diferentes e individuales, donde los alumnos tienen que ir rotando. Estas prácticas se realizan de forma secuencial, independientemente de la materia teórica que en ese momento se esté impartiendo. Esta metodología de organización del laboratorio tiene un coste inferior, ya que en este caso basta con comprar una unidad del material de cada práctica; sin embargo, sólo podría ser verdaderamente efectiva si todas las prácticas se impartiesen una vez finalizadas las clases teóricas, y no de forma simultánea, tal y como se plantea en la mayoría de las planificaciones docentes universitarias (al menos así ocurre en los centros de nuestra universidad donde impartimos docencia).

Por otro lado, sucede que casi siempre los trabajos experimentales propuestos por los fabricantes de material didáctico emplean nomenclaturas y material formativo distintos a los utilizados por el profesor en clases de teoría, y en la mayoría de las ocasiones emplean software propietario de simulación, adquisición de datos y de control. A veces es más complicado estudiar las herramientas y los manuales que suministran, que asimilar los propios fundamentos teóricos que se desean transmitir, lo que requiere también un trabajo extra de traducción y readaptación de los manuales y guiones de prácticas.

Nuestro enfoque es distinto. Pensamos que lo mejor es que el alumno aplique inmediatamente los conceptos que se le transmiten cada semana en la teoría. Para ello, si, por ejemplo, los grupos de prácticas son de 20 alumnos, será necesario disponer al menos de 10 unidades idénticas de material experimental para cada sesión. Evidentemente, la única forma de implantar este sistema, con las dificultades presupuestarias actuales, es abaratando al máximo los costes de las maquetas de control a utilizar, y realizando entre profesores y alumnos la totalidad del trabajo de preparación de material, herramientas de control y guiones de prácticas, para no tener que pagar ese valor añadido a empresas de material didáctico. Es un esfuerzo grande, requiere tiempo, pero pensamos que merece la pena, ya que además si los alumnos 
intervienen directamente en el diseño y construcción de las maquetas que van a utilizar en sus prácticas, se van a involucrar totalmente y aprenderán mucho más.

El laboratorio que se está construyendo, está ya dando servicio a las siguientes asignaturas impartidas en la Universidad de Granada:

- Ingeniería de Sistemas (Grado en Ingeniería Electrónica Industrial)

- Mecatrónica y Sistemas Aeroespaciales (Master de Ciencia de Datos e Ingeniería de Computadores).

- Sistemas de Control (Grado en Ingeniería de Tecnologías de Telecomunicación)

- Automática (Grado en Ingeniería Electrónica Industrial)

- Informática Industrial (Grado en Ingeniería Informática)

- Controladores Lógicos Programables (Grado en Ingeniería Informática)

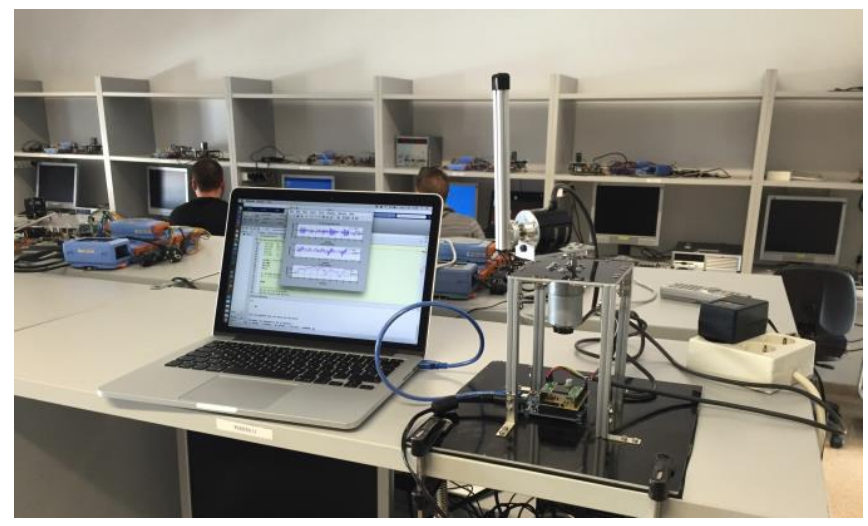

Figura 1. Fotografía parcial del Laboratorio de Control

\section{Objetivos de implementación y de aprendizaje}

A continuación se muestra una descripción de los sistemas que se están desarrollando y los objetivos de aprendizaje a conseguir con la utilización de los mismos.

\subsection{Objetivos de implementación}

En un principio nos hemos planteado la realización de un conjunto de maquetas de control, ya clásicas y habitualmente conocidas, cuyos modelos físicos (en forma de ecuaciones diferenciales, funciones de transferencia o en su representación en el espacio de estados) son fáciles de calcular, y de hecho dichos desarrollos se llevan a cabo en las clases teóricas. Presentamos a continuación una lista inicial: 
- Control de posición (servo) y de velocidad angular de motor de corriente continua.

- Bola en barra.

- Péndulo invertido rotatorio (péndulo de Furuta).

- Péndulo invertido lineal en carrito.

- Mini-Segway.

- Bola en plano.

En la Figura 2 presentamos fotografías de algunos de los primeros prototipos ya realizados:

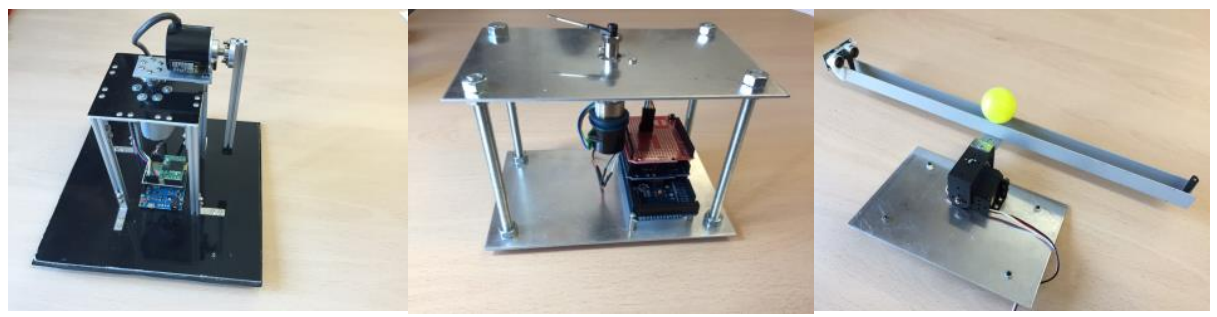

Figura 2. Fotografías de algunas de las maquetas realizadas (péndulo rotativo, servo-motor y bola en barra).

Más adelante continuaremos con más ideas de mecanismos de regulación y control (seguimiento de vehículos eléctricos, segway completo, micro-cuadricópteros, control de mini-trenes, etc.)

\subsection{Objetivos de aprendizaje}

Con este conjunto inicial de sistemas mecatrónicos didácticos, pensamos que es suficiente para que el estudiante comprenda y pueda utilizar las principales técnicas de modelado y de control básico [4], tales como:

- Técnicas de obtención de modelos lineales de sistemas físicos mediante funciones de transferencia y representación en el espacio de estados.

- Control PID (Proporcional-Integral-Diferencial)

- Control digital por ubicación de polos y realimentación en el espacio de estados:

- Configuración con ganancia previa

- Configuración "servo"

- Configuración con integrador

- Controlador-Observador.

- Control LQR (Regulador Cuadrático Lineal).

- Control LQG (Regulador Cuadrático Gaussiano). 
Por otro lado, transversalmente el alumno adquiere conocimientos relacionados con:

- Procedimientos de medida y de cálculo numérico de multiples magnitudes (posición lineal, velocidad lineal, posición angular, velocidad angular, aceleración, detección de posición,...), utilizando sensores de bajo coste (codificadores (encoders) incrementales, ultrasonidos, hilo resistivo, sensores de infrarrojos, cámaras de video, acelerómetros, giróscopos, magnetómetros, pantallas resistivas, etc.) (ver Figura 3).

- Control sobre actuadores (motores de corriente continua y servos).

- Configuración, diseño y desarrollo de sistemas basados en microcontroladores.

- Programación en Matlab-Simulink .

- Programación en tiempo real en lenguaje C.

- Procesamiento digital de señales.

- Comunicaciones.

- Diseño CAD.

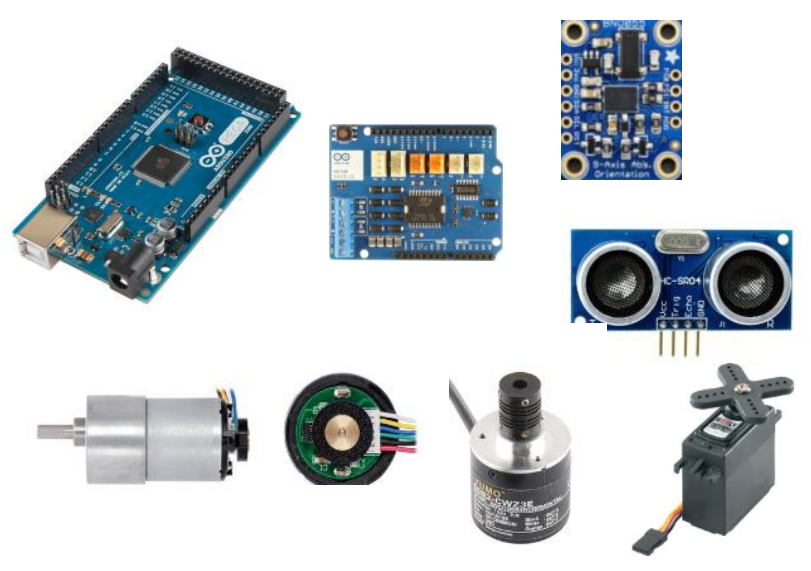

Figura 3. Algunos de los sensores, actuadores y tarjetas de control empleadas.

\section{Metodología de desarrollo empleada}

Considerando que el objetivo primordial es conseguir que el estudiante comprenda mejor los fundamentos de control mediante la práctica experimental, y teniendo en cuenta que antes de acudir al laboratorio, se supone que ya domina Matlab-Simulink como herramienta de simulación, pensamos que es ideal que pueda seguir usando la misma herramienta también para realizar el control en tiempo real de los sistemas mecatrónicos desarrollados. Afortunadamente Mathworks suministra un conjunto de modelos Simulink [5] para Arduino [6] (y también para Raspberry Pi [7]). Este grupo de modelos incluye: lectura y escritura digital y analógica, PWM y comunicaciones por puertos serie, Ethernet y Wifi. Para nuestras aplicaciones didácticas, necesitamos disponer también de bloques de lectura de sensores de distancia mediante 
ultrasonidos, de encoders y de unidades inerciales (IMUs); para ello se deberán usar funciones-S específicas.

Para bajas frecuencias de muestreo y ejemplos sencillos se puede usar el modo "External", que permite la visualización de resultados en tiempo real mediante módulos tales como Scope y Display; pero en nuestro caso, se requieren periodos de muestreo del orden de $\mathrm{T}=0.01 \mathrm{seg}$, y esta opción no se puede utilizar. Es necesario usar el modo "Deploy to hardware" que transfiere totalmente el firmware a la tarjeta de control. Para visualizar los gráficos dinámicos con las variables (estados, salidas y señal de control) hay que incluir bloques de comunicaciones, y realizar después una aplicación Matlab para la lectura y visualización.

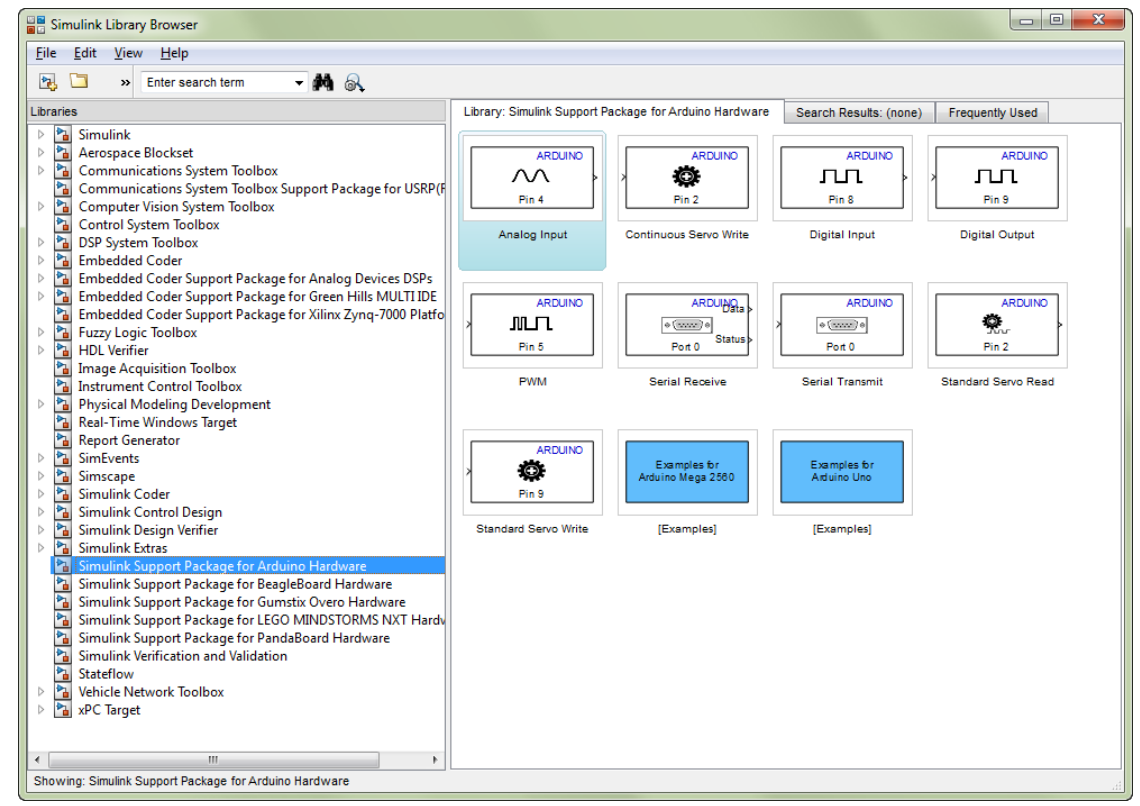

Fig. 4. Módulos de la Biblioteca Arduino para Simulink

El desarrollo de aplicaciones de control con este sistema es muy rápido; sin embargo, el alumno puede pensar que sigue realizando algo muy parecido a la simulación (aunque vea funcionar la maqueta) y es por ello, que pensamos que, alternativamente, es también conveniente que realice por sí mismo todo el software de control.

Se desarrolla también la aplicación directamente en lenguaje C, para que el alumno pueda ver también cómo se estructura una aplicación firmware en tiempo real, y aprenda también a manejar las interrupciones, realizar una derivada discreta en forma precisa (para obtener una velocidad angular a partir de la salida de un encoder, por ejemplo), a leer los pulsos de dicho codificador bajo interrupciones, a realizar un filtro digital con ecuaciones en diferencias, a linealizar la respuesta de control de un motor 
DC (mediante una tabla de "look up"), a manejar la comunicación serie multivariable, etc. Es también muy interesante analizar el firmware en $\mathrm{C}$ que Simulink obtiene cuando se compila una aplicación con sus módulos gráficos. El estudiante puede así compararlo con el programa en $\mathrm{C}$ realizado por él.

Se resumen a continuación las dos configuraciones empleadas.

\subsection{Configuraciones de control}

En un principio proponemos estas dos configuraciones de diseño, control y visualización de resultados:

1. Desarrollo en $\mathrm{C}$ sobre Arduino o Raspberry Pi del software de medida $\mathrm{y}$ control, con comunicación (vía puerto serie USB, Bluetooth, Ethernet o Wifi) con aplicación realizada en Matlab. Se simulan con Matlab los resultados previstos para un diseño de control específico, se visualizan las variables simuladas de estado y de salida, se reciben en tiempo real las magnitudes de cada maqueta y se representan conjuntamente (ver Figura 5).

2. Desarrollo completo en Simulink del firmware de control, que se compila y transfiere totalmente al firmware de las tarjetas de control (ver Figura 6). Para ello se están utilizando la biblioteca de modelos ofrecida por Mathworks, tanto para Arduino, como para Raspberry Pi antes comentada.
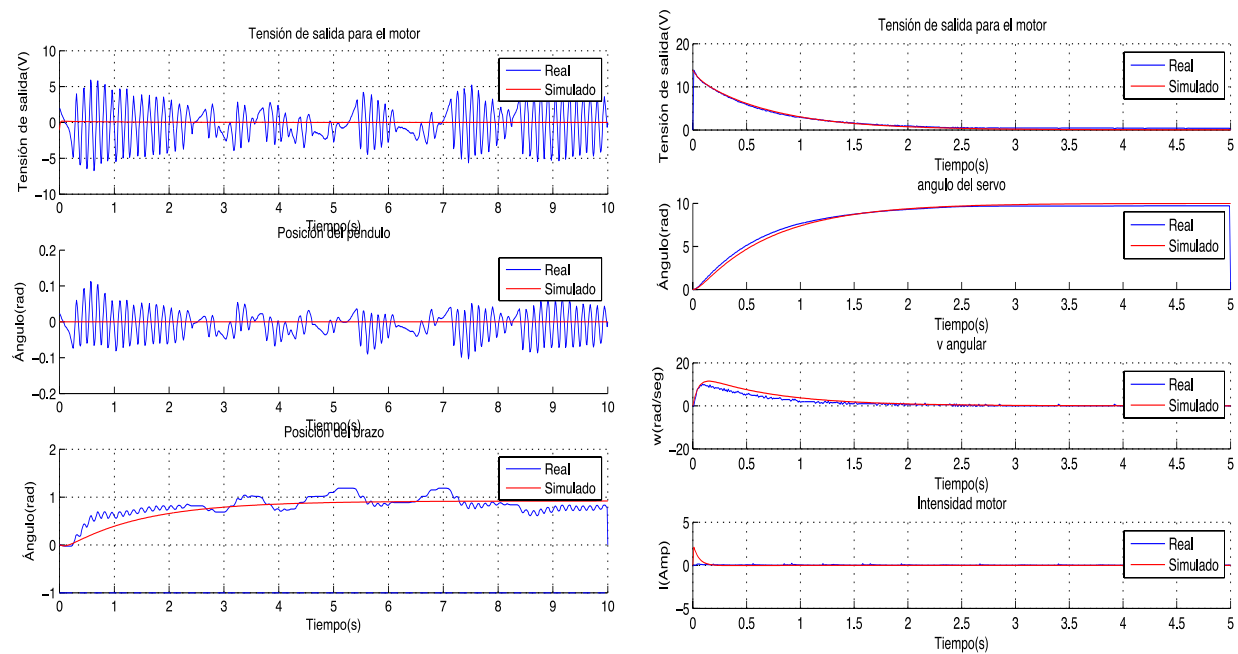

Figura 5. Resultados experimentales y simulados para péndulo invertido y servomotor, con configuración Matlab + firmware en C sobre tarjeta Arduino Mega. 


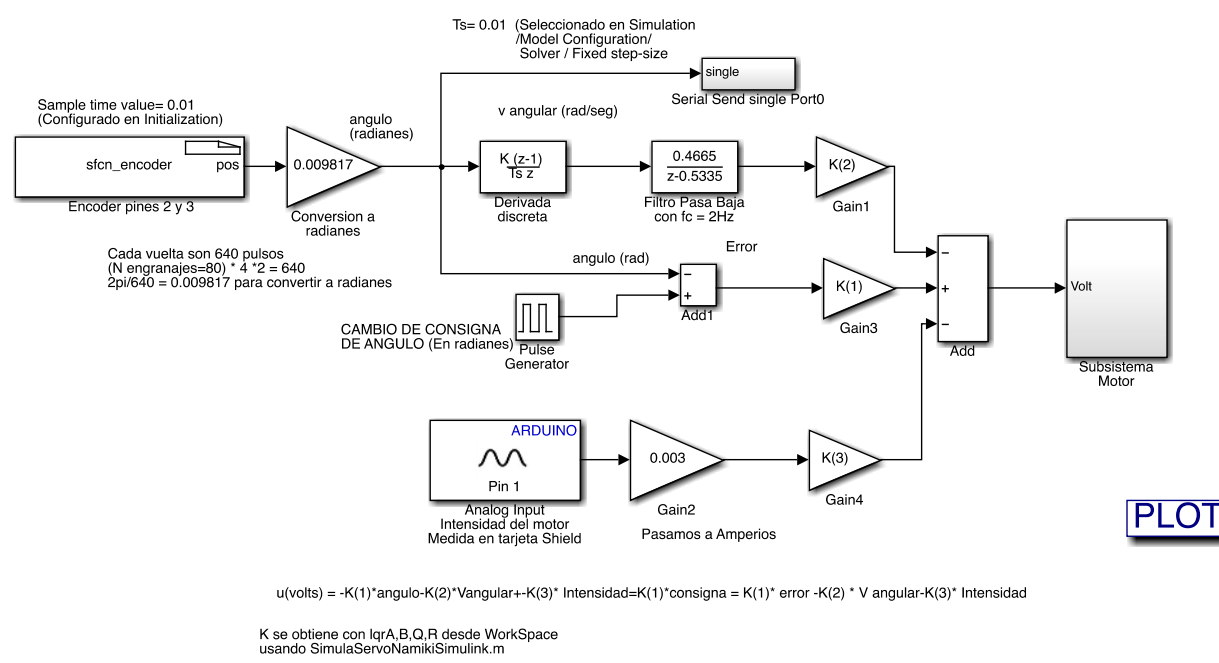

Figura 6. Diseño del control LQR de un servo motor, utilizando la biblioteca de Arduino para Simulink.

Más adelante se tiene previsto utilizar también Labview (National Instruments)[8] con tarjetas RIO, y comunicar los sistemas de control experimental con aplicaciones de móviles tanto para Android como para iOS. Esta solución permite usar los recursos de Labview para visualización en tiempo real, sin necesidad de usar módulos de comunicación a medida; sin embargo, en este caso el coste de las tarjetas de control es mucho mayor. No obstante, también puede conectarse Labview a tarjetas Arduino y Raspberry, y National Instrument permite que los alumnos utilicen Labview durante un año, sin coste alguno.

Actualmente estamos empezando a usar Raspberry Pi para proyectos de control que requieren procesamiento de imágenes, tal como el dispositivo "Bola en plano". La posición de la bola en plano se puede medir en tiempo real con una pantalla resistiva, pero también se puede realizar con una sencilla cámara web, procesando digitalmente la imagen. Este procesamiento de puede realizar fácilmente utilizando los modelos apropiados de la biblioteca de procesamiento de imágenes de Simulink.

Hasta ahora, ninguna de las maquetas realizadas tiene un coste de material superior a $150 €$, y la mayoría rondan los $50 €$, sumando todos los elementos (sensores, actuadores, módulos de potencia, tarjetas de control, mecanización), siempre que ese material se compre directamente vía web a distribuidores de bajo coste, por lo que, sin duda pensamos que los objetivos iniciales se están cumpliendo. 


\section{Conclusiones}

Se ha presentado en este artículo el procedimiento de desarrollo de un laboratorio de control de bajo coste. Las dos configuraciones empleadas: firmware en $\mathrm{C}$ con Matlab frente a Firmware con Simulink son complementarias. Está claro que es más fácil y rápido trabajar en un entorno gráfico, donde gran parte de los modelos ya están disponibles, pero si queremos que el alumno sepa exactamente como se realizan los algoritmos de control en tiempo real, es necesario al menos mostrar las aplicaciones realizadas en $\mathrm{C}$, con la ventaja añadida de poder comparar los resultados simulados con los experimentales. El bajo coste de los materiales utilizados permite la fabricación a mayor escala de las maquetas de prácticas implementadas, por lo que, sin duda, a corto plazo los alumnos podrán disponer del material adecuado para simultanear las prácticas con la teoría.

Agradecimientos. Al Secretariado de Innovación Docente de la Universidad de Granada por los Proyectos de Innovación Docente concedidos que nos han permitido financiar la adquisición de algunas de las maquetas de laboratorio utilizadas en prácticas.

\section{Referencias}

1. M.Damas, G.Olivares, H.Pomares, A.Olivares: "Laboratorio Virtual basado en Web para el ciclo completo de desarrollo de sistemas de control industriales". Workshop en Informática Industrial (WIIND), ISBN: 84-692-2381-9, Universidad Rey Juan Carlos, Madrid, 2009.

2. M.Damas, H.Pomares, G.Olivares: "Innovación en la docencia práctica de asignaturas relacionadas con la informática industrial". $1^{\text {a }}$ Jornadas Andaluzas de Innovación Docente Universitaria. ISBN: 978-84-692-7263-3, Córdoba, 2009.

3. M.Damas, O.Baños, G.Olivares, F.Gómez: "Complementos para Informática Industrial del perfil de Ingeniería de Computadores del Grado de Informática de la UGR: Controladores Lógicos Programables". Enseñanza y Aprendizaje de Ingeniería de Computadores. Revista de Experiencias Docentes en Ingeniería de Computadores. ISSN: 2173-8688, No3, pp.107$120,2013$.

4. Ogata,K. "Ingeniería de Control Moderna". Ed.Prentice Hall. ISBN: 9788483226605, 2010

5. Arduino support from Simulink. http://es.mathworks.com/hardware-support/arduinosimulink.html. Accedido el 4/04/2016.

6. Arduino. https://www.arduino.cc. Accedido el 11/04/2016.

7. Raspberry Pi. https://www.raspberrypi.org/. Accedido el 11/04/2016.

8. National Instruments. https://www.ni.com. Accedido el 11/04/2016. 